\section{Response to An Expanding Role of Biomarkers in Acute Aortic Syndromes}

Pepe G, et al. Clin Cardiol. 2006;29:432-433.

\section{To the Editor:}

Pepe et $\mathrm{a}^{1}$ concluded that the use of genetic and biochemical biomarkers in acute aortic syndromes could be a tool for optimizing the management of this high-risk group of patients. They also show the relevant role of genetic biomarkers in early diagnosis of familial disorders like Marfan syndrome, aortic thoracic aneurysm/dissection, Ehlers-Danlos, and osteogenesis imperfecta syndrome. We believe these biomarkers will be guides to future clinical diagnoses and accurate diagnosis and prognosis of cardiovascular diseases, and for that reason we would like to mention the relevant role of variants that are classified as variants of unknown significance (VUS) in Mendelian disorders such Marfan syndrome. Genetic testing may benefit patients, at-risk family members, and individuals with borderline phenotypes, as well as improve genetic counseling and allow critical differential diagnoses. ${ }^{2} \mathrm{We}$ have reported 2 family cases with 4 and 7 family members genetically analyzed for the $F B N 1$ gene. Some of them were discovered to be affected by 2 VUS not previously described in published data or in databases such as The Human Gene Mutation Database-FBN1 Mutations Database (http://www.hgmd.cf.ac.uk/ac/search.php) or Genecards (http://www.genecards.org) and UniProt (http://www.uniprot.org/).

After genetic analysis of these familial cases, we confirmed that VUS mutations p.Cys2659Stop (p.C2659X) and p.Gln1419Stop (p.Q1419X) at (c.4255 C>Y) conferred a predisposition to Marfan syndrome. Two members of a family presented the C2659X mutation, and 4 presented the Q1419X mutation; all of them were confirmed by the doctor to be affected by Marfan syndrome. To confirm the effect of these variants, we performed bioinformatics analysis with PolyPhen-2 (http://genetics.bwh.harvard.edu/ pph2/), SNPsGO (http://snps-and-go.biocomp.unibo.it/ snps-and-go), MutPred (http://mutpred.mutdb.org), SIFT (http://sift.jcvi.org), and Align-GVGD (http://agvgd.iarc. fr/agvgd_input.php) software. Furthermore, nonsense mutations such as C2659X have a pathogenic effect on their own in most cases and in this particular case. In both of these cases, carriers have a shortened final protein. Even in p.Q1419X there is a 50\% reduction of the normal amino acid residue.

This genetic analysis has conferred a better medical follow-up to all of these family members, which is relevant for improving their quality of life and that of their descendants, who can enjoy this medical advantage. With these described clinical and genetic cases, we aim to include these variants in the FBN1 gene as having a relevant role in Marfan syndrome so that other patients and medical personnel can have these clinical advantages.

In conclusion, our report on both of these familial cases with Marfan syndrome and the presence of C2659X and Q1419X variants in the $F B N 1$ gene, that until now was unclassified as pathogenic, could be suggested to have a pathogenic effect by the presence in many family members, most of them affected by this syndrome. These mutations could be suggested in the framework of genetic counseling as pathogenic, with the combination of other clinical parameters evaluated as Ghent criteria and Marfan syndrome clinical diagnosis criteria. This mutation and the screening in FBN1, TGFBR1, and TGFBR2 genes could encourage individuals in families with pathogenic variants to participate in an intensified screening program similar that in BRCA1/2 in breast cancer ${ }^{3} ; M L H 1, M S H 2$, and $M S H 6$ in colorectal cancer ${ }^{4}$; and others. We suggest that molecular genetics biomarkers can be useful tools in identifying subjects at risk of developing cardiac diseases such as aortic aneurysms, acute aortic syndrome, or Marfan syndrome, as well as for screening for early symptoms. We believe that having updated information of the main polymorphisms in $F B N 1, T G F B R 1$, and TGFBR2 genes is necessary.

Fernando Cabrera-Bueno, MD, $\mathrm{PhD}$ Cardiology Department, Section of Marfan Universitary Hospital "Virgen de la Victoria" Malaga, Spain

Maria Jesus Alvarez-Cubero, $\mathrm{PhD}$ GENYO (Pfizer-University of Granada Andalusian Government Centre for Genomics and Oncological Research) Granada, Spain Francisco Fernandez-Rosado, PhD Esther Martinez-Espin, $\mathrm{PhD}$ Carmen Entrala-Bernal, $\mathrm{PhD}$

Lorgen G.P, S.L., Business Innovation Center - BIC/CEEL, Technological area of Health Science, Av. de la Innovación 1 Granada 18016, Spain

\section{References}

1. Pepe G, Giusti B, Yacoub M. An expanding role of biomarkers in acute aortic syndromes. Clin Cardiol. 2006;29:432-433.

2. McInerney-Leo AM, Marshall MS, Gardiner B, et al. Whole exome sequencing is an efficient, sensitive and specific method of mutation detection in osteogenesis imperfecta and Marfan syndrome. Bonekey Rep. 2013;2:456.

3. Guinan EM, Hussey J, McGarrigle SA, et al. A prospective investigation of predictive and modifiable risk factors for breast cancer in unaffected BRCA1 and BRCA2 gene carriers. BMC Cancer. 2013;13:138.

4. Rahner N, Steinke V, Schlegelberger B, et al. Clinical utility gene card for: Lynch syndrome (MLH1, MSH2, MSH6, PMS2, EPCAM) - update 2012 [published online ahead of print August 15, 2012]. Eur J Hum Genet. doi: 10.1038/ejhg.2012.164. 